\title{
Is Omentectomy Mandatory Among Early Stage (I, II) Malignant Ovarian Germ Cell Tumor Patients? A Retrospective Study of 223 Cases
}

\author{
Wenyan $X u, M D$ and Yanfang $L i, M D$
}

\begin{abstract}
Objective: The aim of the study was to investigate whether omentectomy (OMT) is necessary in the operation for apparently early stage malignant ovarian germ cell tumors (MOGCTs). Methods and Materials: Searching medical records database of Sun Yat-sen University Cancer Center from January 1, 1966, to November 30, 2015, patients with MOGCTs were identified and their age, year of diagnosis, tumor grade, histologic subtype, International Federation of Gynecology and Obstetrics stage, nodal findings, gross observation of omentum, and performance of OMT were assessed. Overall survivals of patients with or without OMT were compared using Kaplan-Meier survival curves.

Results: A total of 223 MOGCT cases with clinically early stage (stage I and II) disease and with the 3 common histological subtypes of MOGCT were obtained, which include yolk sac tumor (YST), dysgerminoma (DSG), and immature teratoma (IMT). There were 192 stage I cases and 31 stage II cases. Fifty-four patients were diagnosed with YST, 61 with DSG, and 108 with IMT. Omentectomy was performed as part of the initial surgery in $74.0 \%$ patients $(165 / 223)$ and was omitted in $26.0 \%$ patients $(58 / 223)$. Chemotherapy was administered in $88.3 \%(197 / 223)$ of all patients. The median follow-up was 82.0 months. The 10-year overall survival rates of the patients with and without OMT were $90.5 \%$ and $98.1 \%$, respectively $(P=0.156)$. Regarding different stages or histological subtypes, the 10-year survival rates of the 2 groups were $92.0 \%$ versus $97.9 \%$ ( $P=0.324$, stage $\mathrm{I}), 83.2 \%$ versus $100 \%(P=0.351$, stage II), $89.2 \%$ versus $100 \%(P=0.303$, YST $), 94.1 \%$ versus $100 \%$ ( $P=0.470$, DSG), and $89.4 \%$ versus $96.0 \%(P=0.405$, IMT), respectively.

Conclusions: In conclusion, OMT in patients with clinically early stage MOGCT may not improve patient survival and may be omitted.
\end{abstract}

Key Words: Malignant ovarian germ cell tumor, Omentectomy, Clinically early stage

Received February 8, 2017, and in revised form May 14, 2017.

Accepted for publication March 21, 2017.

(Int J Gynecol Cancer 2017;27: 1373-1378)

$\mathrm{O}$ varian cancer is the most common cause of death from gynecologic malignancy. Malignant germ cell tumors (MOGCTs) account for approximately $15 \%$ of ovarian cancer and occur more frequently in teenage girls or young women. Immature teratoma (IMT), dysgerminoma (DSG), and yolk sac tumor (YST) are the 3 common types of MOGCT. Since
Department of Gynecologic Oncology, Sun Yat-sen University Cancer Center; State Key Laboratory of Oncology in South China; Collaborative Innovation Center for Cancer Medicine, Guangzhou, People's Republic of China.

Copyright (C) 2017 The Author(s). Published by Wolters Kluwer Health, Inc. on behalf of IGCS and ESGO. This is an open-access article distributed under the terms of the Creative Commons Attribution-Non ISSN: $1048-891 \mathrm{X}$

DOI: $10.1097 /$ IGC.0000000000001012
Commercial-No Derivatives License 4.0 (CCBY-NC-ND), where it is permissible to download and share the work provided it is properly cited. The work cannot be changed in any way or used commercially without permission from the journal.

Address correspondence and reprint requests to

Yanfang Li, MD, Department of Gynecologic Oncology,

Sun Yat-sen University Cancer Center, 651 Dongfeng Rd E,

Guangzhou, People's Republic of China, 510060.

E-mail: liyf@sysucc.org.cn.

The authors declare no conflicts of interest. 
1970 s, the introduction of platinum-based chemotherapy into the treatment of MOGCT has dramatically increased the overall survival of patients. Now, the standard treatment of MOGCT is usually surgery followed by chemotherapy of BEP regimen (bleomycin, etoposide, and cisplatin). Currently, initial surgical style is mostly fertility-sparing surgery and comprehensive staging, which includes also omentectomy (OMT) in fertility-desired patients or comprehensive staging surgery in fertility not desired patients.

However, is OMT really necessary in early stage MOGCT? Recently, in clinically apparent early stage epithelial ovarian cancer, the significance of OMT was doubted. For epithelial ovarian cancer, extraovarian spread disease confined to the omentum is found in $2 \%$ to $7 \%$ of cases at most. ${ }^{1}$ For patients without visible omental disease and for whom adjuvant chemotherapy is planned, OMT seems to be mainly for role of staging, although its therapeutic role remains unclear in microscopic omental disease. ${ }^{1}$ As for MOGCT, few researches focused on this issue. One study recently suggested that staging surgery, in which OMT was included, for patients with apparently early stage MOGCT should be omitted. ${ }^{2}$ Previously, our data in 45 patients with stage I or II YST showed that OMT might not improve patient survival. ${ }^{3}$ In this study, we aim to further investigate the role of OMT on survival of patients with apparently early stage MOGCT.

\section{MATERIALS AND METHODS}

We retrospectively reviewed all cases with MOGCT treated at Sun Yat-sen University Cancer Center from January 1, 1966, to November 30, 2015. Approval for this study was granted by Sun Yat-sen University Cancer Center's Human Research Protection Office (YB2015-033-01). These cases were identified through hospital databases. Only patients diagnosed with IMT, DSG, and YST and with stage I to II disease were included. The patients' medical records were reviewed to collect case information including age, surgical procedures, operative findings, histopathology, International Federation of Gynecology and Obstetrics (2013) stage, adjuvant chemotherapy, and follow-up.

The software SPSS was used to analyze the data. We use the Kaplan-Meier method to calculate the survival rate. Overall survival was defined from the date of initial surgery to the time to death or last follow-up. When comparing the baseline of non-OMT (NOMT) and OMT groups, we used analysis of variance for age and cross-table method for the rest factors. A $P$ value of less than 0.05 was considered statistically significant.

\section{RESULTS}

\section{Patient Characteristics}

There are 457 patients who were diagnosed with the 3 types of MOGCT mentioned previously at our cancer center from January 1, 1966, to November 30, 2015. Among them, a total of 223 patients were diagnosed with early stage (stage I to II) disease, including 192 stage I cases and 31 stage II. The median (range) age was 25 (8-67) years. Fifty-four patients were diagnosed with YST, 61 with DSG, and 108 with IMT. Patient characteristics are listed in Table 1. Of all 223 patients, $165(74.0 \%)$ received OMT as part of surgical procedures and $58(26.0 \%)$ did not during the initial surgery. On the basis of OMT performed or not, we divided the patients into 2 groups. The following factors in the 2 groups were compared: age, stage, pathological subtype, other surgical procedure (hysterectomy or pelvic lymphadenectomy), and platinum-based chemotherapy. The mean age was 27.4 and 24.1 in patients with and without OMT, respectively. Of the group with OMT, 139 patients $(84.2 \%)$ had stage I disease and $26(15.8 \%)$ stage II; of the group without OMT, 53 patients $(91.4 \%)$ had stage I disease and $5(8.6 \%)$ stage II. As of tumor type, the number of patients with YST, DSG, or IMT were $43(26.0 \%)$, $44(26.7 \%)$, and $78(47.3 \%)$, respectively, in the OMT group and that was $11(19.0 \%), 17(29.3 \%)$, and $30(51.7 \%)$, respectively, in the group without OMT. Regarding adjuvant chemotherapy, 139 patients $(84.2 \%)$ received platinum-based regimens in OMT group and $46(79.3 \%)$ in the group without OMT. There was no significant difference in terms of age,

TABLE 1. Patient characteristics $(N=223)$

\begin{tabular}{|c|c|}
\hline Parameter & n $(\%)$ \\
\hline Age, median (range), y & $25(8-67)$ \\
\hline \multicolumn{2}{|l|}{ Stage } \\
\hline I & $192(86.1)$ \\
\hline I, NOS & $25(11.2)$ \\
\hline IA & $97(43.5)$ \\
\hline IB & $4(1.8)$ \\
\hline $\mathrm{IC}$ & $66(29.6)$ \\
\hline IC, NOS & $9(4.0)$ \\
\hline IC1 & $30(13.5)$ \\
\hline $\mathrm{IC} 2$ & $24(10.8)$ \\
\hline IC3 & $3(1.4)$ \\
\hline II & $31(13.9)$ \\
\hline II, NOS & $11(4.9)$ \\
\hline IIA & $2(1.0)$ \\
\hline IIB & $18(8.1)$ \\
\hline \multicolumn{2}{|l|}{ Histopathology } \\
\hline YST & $54(24.2)$ \\
\hline Stage I & $46(20.6)$ \\
\hline Stage II & $8(3.6)$ \\
\hline DSG & $61(27.4)$ \\
\hline Stage I & $54(24.2)$ \\
\hline Stage II & $7(3.1)$ \\
\hline IMT & $108(48.4)$ \\
\hline Stage I & $92(41.3)$ \\
\hline Stage II & $16(7.2)$ \\
\hline
\end{tabular}

NOS, not otherwise specified, we cannot clarify the specific stage from the data we got. 
TABLE 2. Characteristics of the 2 groups: OMT done versus not done

\begin{tabular}{lccc}
\hline Parameter & $\begin{array}{c}\text { OMT Done } \\
(\mathbf{n}=\mathbf{1 6 5})\end{array}$ & $\begin{array}{c}\text { OMT Not } \\
\text { Done }(\mathbf{n}=\mathbf{5 8})\end{array}$ & $\boldsymbol{P}$ \\
\hline $\begin{array}{l}\text { Mean age, y } \\
\text { Stage, (\%) }\end{array}$ & 27.4 & 24.2 & 0.085 \\
$\quad$ I & $139(72.4)$ & $53(27.6)$ & 0.177 \\
II & $26(83.9)$ & $5(16.1)$ & \\
$\quad \begin{array}{l}\text { Tumor type, (\%) } \\
\quad \text { YST }\end{array}$ & $43(79.6)$ & $11(20.4)$ & 0.278 \\
DSG & $44(72.1)$ & $17(27.9)$ & 0.698 \\
$\quad$ IMT & $78(72.2)$ & $30(27.8)$ & 0.560 \\
$\begin{array}{l}\text { Surgical procedure, (\%) } \\
\quad \text { Hysterectomy }\end{array}$ & $59(35.8)$ & $5(8.6)$ & $<0.001$ \\
$\quad$ Pelvic & $79(47.9)$ & $3(5.2)$ & $<0.001$ \\
$\quad$ lymphadenectomy & & & \\
$\quad \begin{array}{l}\text { Lymph node biopsy } \\
\text { Platinum-based } \\
\text { chemotherapy, (\%) }\end{array}$ & $7(4.2)$ & $2(3.5)$ & 1.000 \\
& & $46(79.3)$ & 0.390 \\
& & & \\
\hline
\end{tabular}

stage distribution, tumor type, and the percentage of platinumbased chemotherapy between the 2 groups (all $P>0.05$ ).

Patients who received OMT were more likely to have hysterectomy and pelvic lymphadenectomy $(35.8 \%$ vs $8.6 \%$ and $47.9 \%$ vs $5.2 \%$, respectively, both $P$ values $<0.05$ ) (Table 2 ).

\section{Survival Analysis}

The median follow-up time was 82.0 months. The 5 - and 10-year overall survival rates for the 223 patients were $93.7 \%$ and $92.5 \%$, respectively. Fourteen patients died and 209 survive up till now. The 5-year survival rates for patients with and without OMTwere $92.2 \%$ and $98.1 \%$, respectively $(P=0.174)$. The 10 -year survival rates for the 2 groups were $90.5 \%$ and $98.1 \%$, respectively $(P=0.156)$ (Table 3; Figs. 1A, B).

Among patients with stage I disease, 139 (72.4\%) received OMT whereas $53(27.6 \%)$ did not. The 5- and 10-year survival rates for patients with and without OMT were $94.1 \%$ versus $97.9 \%(P=0.366)$ and $92.0 \%$ versus $97.9 \%(P=0.324)$, respectively. Of the 31 stage II patients, $26(83.9 \%)$ received OMT and $5(16.1 \%)$ did not. The 5-year survival rates for patients with and without OMT were $83.2 \%$ and $100 \%(P=$ $0.351)$, respectively. The 10 -year survival rates for the 2 groups were also $83.2 \%$ and $100 \%(P=0.351)$, respectively (Figs. 1C, D).

Among 54 patients with YST, 43 (79.6\%) received OMT and $11(20.4 \%)$ did not. The 5-year survival rates for patients with and without OMT were $89.2 \%$ and $100 \%$, respectively $(P=0.303)$, and the 10 -year survival rates for the 2 groups were also $89.2 \%$ and $100 \%$, respectively $(P=0.303)$. Likely, of 108 patients with IMT, $78(72.2 \%)$ received OMT and $30(27.8 \%)$ did not. The 5-year survival rates for patients with and without OMT were $89.4 \%$ and $96.0 \%$, respectively $(P=0.405)$; the 10 -year survival rates for the 2 groups were the same as the 5-year survival rate. As for the 61 DSG patients, 44 (72.1\%) had OMT and $17(27.9 \%)$ did not. None of them died in 5 years after initial surgery. Both groups' 5-year survival rate was $100 \%$.

TABLE 3. Survival rate of patients with and without OMT as of different stages and histological subtypes

\begin{tabular}{|c|c|c|c|c|}
\hline Group & $\begin{array}{c}5-y \\
\text { Survival Rate }\end{array}$ & $P$ & $\begin{array}{c}10-y \\
\text { Survival Rate }\end{array}$ & $P$ \\
\hline Total & 93.7 & - & 92.5 & - \\
\hline OMT & 92.2 & $P=0.174$ & 90.5 & $P=0.156$ \\
\hline NOMT & 98.1 & & 98.1 & \\
\hline Stage I & 95.1 & - & 93.7 & - \\
\hline OMT & 94.1 & $P=0.366$ & 92.0 & $P=0.324$ \\
\hline NOMT & 97.9 & & 97.9 & \\
\hline Stage II & 86.0 & - & 86.0 & - \\
\hline OMT & 83.2 & $P=0.351$ & 83.2 & $P=0.351$ \\
\hline NOMT & 100.0 & & 100.0 & \\
\hline YST & 91.4 & - & 91.4 & - \\
\hline OMT & 89.2 & $P=0.303$ & 89.2 & $P=0.303$ \\
\hline NOMT & 100.0 & & 100.0 & \\
\hline DSG & 100.0 & - & 96.0 & - \\
\hline OMT & 100.0 & None & 94.1 & $P=0.470$ \\
\hline NOMT & 100.0 & & 100.0 & \\
\hline IMT & 91.2 & - & 91.2 & - \\
\hline OMT & 89.4 & $P=0.405$ & 89.4 & $P=0.405$ \\
\hline NOMT & 96.0 & & 96.0 & \\
\hline
\end{tabular}



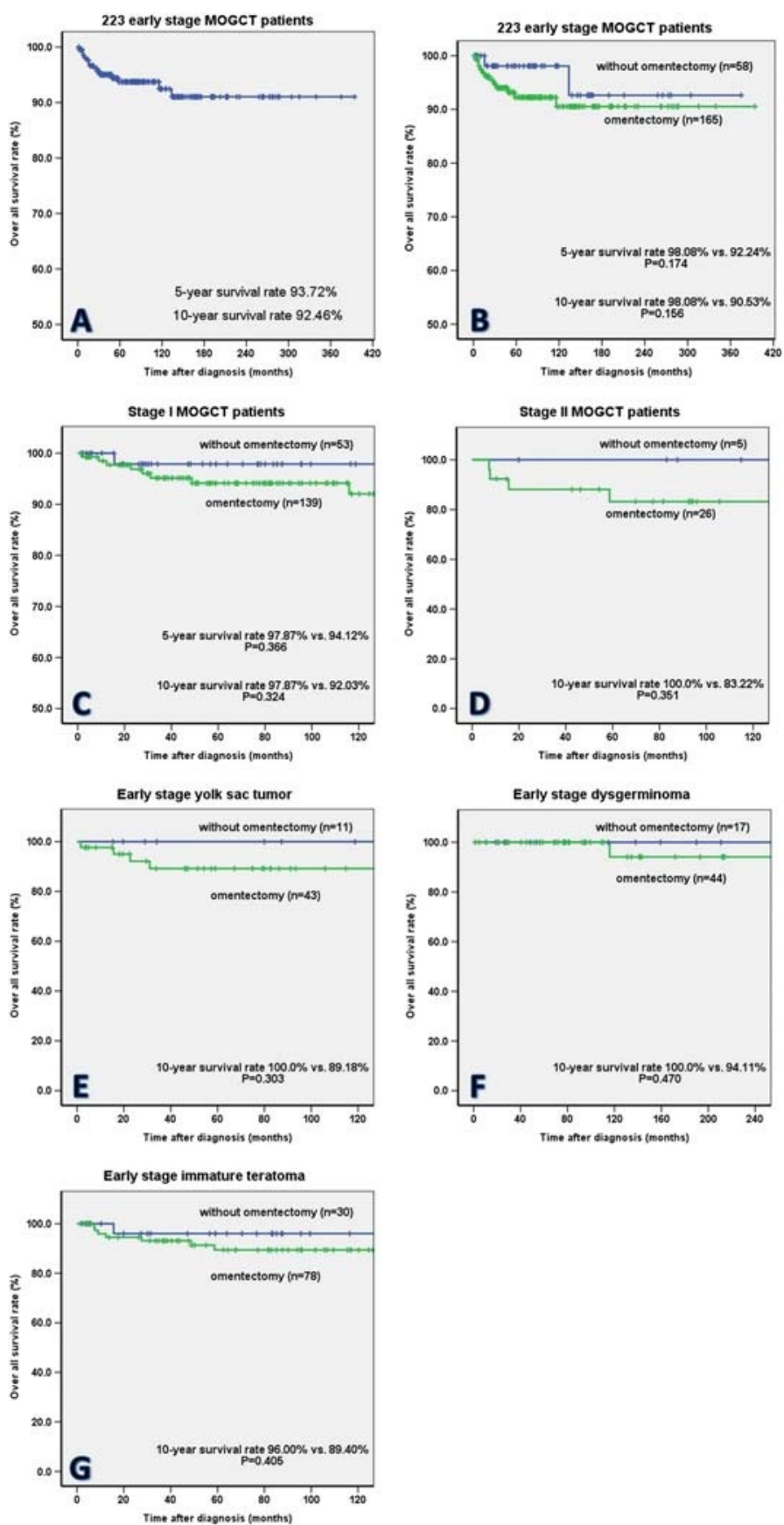

FIGURE 1. Survival rate of 223 patients with early stage MOGCT. A, The 5- and 10-year survival rates of the 223 patients with early stage MOGCT were $93.72 \%$ and $92.46 \%$, respectively. B, A total of 165 patients in OMT group and 58 in NOMT group. The 5-year survival rates were $92.24 \%$ and $98.08 \%$, respectively, for the 2 groups $(P=0.174)$. The 10 -year survival rates were $90.53 \%$ and $98.08 \%$, respectively $(P=0.156)$. C, There were 192 stage I patients, $139(72.40 \%)$ of them received OMT while $53(27.60 \%)$ did not. The 5-year survival rates were $94.12 \%$ and $97.87 \%(P=0.366)$, respectively. The 10 -year survival were $92.03 \%$ and $97.87 \%(P=0.324)$, respectively. D. Of the 31 stage II patients, 26 were in OMT group and 5 in NOMT group. The 5- and 10-year survival rates were the same. The OMT group was $83.22 \%$ and the NOMT group was $100 \%(P=0.351)$. E, The 54 YST patients had the same 5- and 10-year survival rate. They were $89.18 \%$ and $100 \%$ for OMT group $(n=43)$ and NOMT group $(n=11)$, respectively $(P=0.303)$. F, None of the 61 DSG patients died in 5 years after initial surgery. Both groups' 5 -year survival rate was $100 \%$. The 10 -year survival rate of OMT group $(n=44)$ was 94.11\% versus $100 \%$ for NOMT group $(n=17)(P=0.470)$. $G$, In 108 IMT patients, the 5 - and 10-year survival rates were the same. It was $89.40 \%$ for OMT group $(n=78)$ and $96.00 \%$ for NOMT group $(n=30)(P=0.405)$. 
TABLE 4. Multivariate analysis for 10-year

overall survival

\begin{tabular}{|c|c|c|c|}
\hline Parameter & $P$ & $\mathbf{R R}$ & $95 \% \mathrm{CI}$ \\
\hline Age ( $\geq 50$ y vs $<50$ y) & 0.005 & 7.262 & $1.824-28.908$ \\
\hline hology & 0.913 & 1.038 & 0.53 \\
\hline Stage (I vs II) & 0.559 & 1.457 & 0.413 \\
\hline es vs no) & 0.405 & 2.566 & 0.279 \\
\hline Hysterectomy (yes vs no) & 0.398 & 1.920 & 0.422 \\
\hline $\begin{array}{l}\text { Pelvic lymphadenectomy } \\
\text { (yes vs no) }\end{array}$ & 0.420 & 0.571 & $0.146-2.229$ \\
\hline $\begin{array}{l}\text { Platinum-based chemotherapy } \\
\text { (yes vs no) }\end{array}$ & 0.420 & 2.375 & $0.290-19.431$ \\
\hline
\end{tabular}

CI, confidence interval; RR, relative risk.

The 10-year survival rates for patients with and without OMT were $94.1 \%$ and $100 \%$, respectively $(P=0.470)$ (Fig. $1 \mathrm{E}-\mathrm{G})$.

We perform a multivariate analysis using COX model to evaluate the impact of different factors on 10-year overall survival in terms of age, histology, stage, OMT, hysterectomy, pelvic lymphadenectomy, and platinum-based chemotherapy. The results revealed that only age is an independent prognostic factor for 10 -year survival rate (Table 4), which also suggested that OMT may not be a prognostic factor.

\section{DISCUSSION}

Our study suggested that in patients with early stage MOGCT performing OMT may not improve patient survival. To our knowledge, this is one of the largest cohorts of patients thus far focusing on investigating the necessity of OMT in apparently early stage MOGCT.

Omentum is a peritoneal organ of different functions. It may play a role in protecting the peritoneal cavity and fighting infection. ${ }^{4}$ Study showed that omentum may help heal injured or inflamed tissue or organ and prevent adhesion formation. ${ }^{5}$ In the situation of cancer from peritoneal organ such as ovarian cancer, omentum may harvest the circulating cancer cells and prevent cell spread to greater extent. ${ }^{5}$

The therapeutic significance of OMT in ovarian cancer remains unclear. In epithelial ovarian cancer, OMT is traditionally recommended and included in the surgery for staging or as a part of tumor cytoreduction in early or advanced stage disease no matter whether there is macroscopic lesion. This is based on the considerations that ovarian cancer is easy to spread in the peritoneal cavity and omentum is the main metastasis site of tumor spread. ${ }^{6}$ However, recently, the necessity of removing grossly normal omentum is doubted in early stage epithelial ovarian cancer. ${ }^{5,7,8}$ Studies showed that the incidence of isolated metastasis to omentum is low $(2 \%-7 \%)$ in patients with stage I and II epithelial ovarian cancer and with a macroscopically normal omentum. The rate is related to tumor stage, grade, and histology. Random biopsies instead of total OMT may be enough for staging purpose in clinically early epithelial ovarian cancer. ${ }^{5} \mathrm{McNally} \mathrm{et} \mathrm{al}{ }^{7}$ showed that OMT did not seem to improve patient survival in a retrospective series of 20,975 cases with stage I to IIIA epithelial ovarian cancer from the Surveillance, Epidemiology, and End Results Database.

In MOGCT, traditionally, complete staging surgery including OMT is also recommended as part of the surgical procedure in early stage disease (National Comprehensive Cancer Network guidlines). ${ }^{6}$ However, few data in the literature are available supporting the rationality of OMT in this early situation. Thus, the prognostic significance of OMT in early stage MOGCT is uncertain. Studies showed that staging surgery, in which OMT is included as part of the procedure, in clinically early stage MOGCT may not improve patient survival compared with nonstaging patients. ${ }^{2,9}$ In the study by Zhao et al, ${ }^{2} 23$ of 102 patients with clinically stage I MOGCT received staging surgery. The results showed that complete staging surgery was not a prognostic factor for disease-free survival. ${ }^{2}$ Liu et $\mathrm{al}^{10}$ analyzed the impact of staging surgery on survival in 92 patients with stage I to IV MOGCT, in which half received staging surgery and half nonstaging. Results showed that the tumor-free 5-year survival rates were $87 \%$ and $97 \%$ in the comprehensive staging surgery and nonstaging groups, respectively $(P=0.115) .{ }^{10}$ In the literature, we did not find study that specially evaluated the usefulness of OMT on prognosis of patient with MOGCT. Our previous report on ovarian YST showed that OMT may not improve patient survival. Of the 66 patients, 45 had stage I and II disease; 36 of 45 patients received OMT during their initial surgery and 9 did not. The 5 -year survival rates for patients with and without OMT were $89.2 \%$ and $100 \%$, respectively $(P>0.05){ }^{3}$ In this study, we also showed that the 5- and 10-year survival of patients with OMT is not higher than that of patients without OMT $(92.4 \%$ vs $98.1 \%, 90.5 \%$ vs $98.1 \%$ ). The results even favor the group without OMT. These results suggested that OMT in MOGCT may not help improve patient survival.

Why OMT did not confer survival improvement to patients with stage I and II MOGCT? First, these clinically early patients might have low incidence of microscopic metastasis to omentum as it is in epithelial ovarian cancer. Because our study did not include stage IIIA patients after pathological diagnosis, we cannot evaluate the occult metastasis rate of the omentum. In the literature, data addressing this issue are rare. In our previous report about ovarian YST, microscopic omental metastasis is found in only $2.7 \%$ patients $(1 / 37)$ with grossly normal omentum. ${ }^{3}$ In the report by Zhao et $\mathrm{al}^{2}$ on 102 patients with MOGCT and with the disease grossly confined to the ovary, none of the 49 patients who received OMT had microscopic metastasis to the omentum. ${ }^{2}$ Second, MOGCT is usually sensitive to chemotherapy, and postoperation chemotherapy is generally planned for MOGCT patients except those with stage IA DSG or IMT. For stage IA DSG or IMT, the frequency of microscopic metastasis to omentum is even lower because of the early disease, and surgical procedure without OMT in this situation is theoretically enough; for other clinically early stage diseases beyond the previous situation, the planned postoperation chemotherapy is possible to control the maybe microscopically metastasis on the omentum in the situation with the omentum left unremoved because the disease has high sensitivity to chemotherapy. Therefore, OMT might not have 
therapeutic significance and might be omitted in patients with clinically early MOGCT.

Two other points should be considered. First, the group with OMT had more patients receiving hysterectomy and pelvic lymphadenectomy as compared with the group without OMT. Hysterectomy, OMT, and pelvic lymphadenectomy are traditionally included in the "staging" surgery of MOGCT. One main argue that more frequency of hysterectomy and pelvic lymphadenectomy in the OMT group may compromise the patient survival. To evaluate the prognostic impact of factors in terms of age, stage distribution, tumor type, chemotherapy, and surgical style on survival, we performed a multivariate analysis using COX model. The results showed that hysterectomy and pelvic lymphadenectomy are not independent prognostic factors. This is consistent to the report by Zhao et $\mathrm{al}^{2}{ }^{2}$ which also suggested that complete staging surgery including hysterectomy and pelvic lymphadenectomy may not improve patient survival.

In conclusion, OMT in patients with clinically early stage MOGCT may not improve patient survival and may be omitted.

\section{REFERENCES}

1. Arie AB, McNally L, Kapp DS, et al. The omentum and omentectomy in epithelial ovarian cancer: a reappraisal: part II - the role of omentectomy in the staging and treatment of apparent early stage epithelial ovarian cancer. Gynecol Oncol. 2013;131:784-790.
2. Zhao T, Zhang H, Liu Y, et al. The role of staging surgery in the treatment of apparent early-stage malignant ovarian germ cell tumours. Aust N Z J Obstet Gynaecol. 2016;56:398-402.

3. Wang X, Ma Z, Li Y. Ovarian yolk sac tumor: the experience of a regional cancer center. Int J Gynecol Cancer. 2016;26: 884-891.

4. Beelen RH. Role of omental milky spots in the local immune response. Lancet. 1992;339:689.

5. Arie AB, McNally L, Kapp DS, et al. The omentum and omentectomy in epithelial ovarian cancer: a reappraisal. Part I-omental function and history of omentectomy. Gynecol Oncol. 2013;131:780-783.

6. NCCN. NCCN Clinical Practice Guidelines in Oncology: Ovarian Cancer, version 1. 2016. June 30, 2016. Available at: https://www.nccn.org/professionals/physician_gls/pdf/ovarian. pdf. Accessed January 27, 2017.

7. McNally L, Teng NN, Kapp DS, et al. Does omentectomy in epithelial ovarian cancer affect survival? An analysis of the Surveillance, Epidemiology, and End Results database. Int J Gynecol Cancer. 2015;25:607-615.

8. Yokoyama Y, Hirakawa H, Wang H, et al. Is omentectomy mandatory in the operation for ovarian cancer? Preliminary results in a rat study. Eur J Obstet Gynecol Reprod Biol. 2012;164:89-92.

9. Mahdi H, Swensen RE, Hanna R, et al. Prognostic impact of lymphadenectomy in clinically early stage malignant germ cell tumour of the ovary. Br J Cancer. 2011;105:493-497.

10. Liu Q, Ding X, Yang J, et al. The significance of comprehensive staging surgery in malignant ovarian germ cell tumors. Gynecol Oncol. 2013;131:551-554. 